\title{
Word length effect in free recall of randomly assembled word lists
}

\author{
Mikhail Katkov' ${ }^{1}$ Sandro Romani ${ }^{2}$ and Misha Tsodyks ${ }^{1,3}$ * \\ ' Department of Neurobiology, The Weizmann Institute of Science, Rehovot, Israel \\ ${ }^{2}$ Center for Theoretical Neuroscience, Columbia University, New York, NY, USA \\ ${ }^{3}$ Nizhny Novgorod Neuroscience Center, Institute of Living Systems, University of Nizhny Novgorod, Nizhni Novgorod, Russia
}

Edited by:

Si Wu, Beijing Normal University, China

Reviewed by:

Alessandro Treves, Scuola

Internazionale Superiore di Studi

Avanzati (SISSA), Italy

Christian Leibold, Ludwig

Maximilians University, Germany

*Correspondence:

Misha Tsodyks, Department of

Neurobiology, The Weizmann

Institute of Science, Rehovot,

76100, Israel

e-mail:misha@weizmann.ac.il
In serial recall experiments, human subjects are requested to retrieve a list of words in the same order as they were presented. In a classical study, participants were reported to recall more words from study lists composed of short words compared to lists of long words, the word length effect. The world length effect was also observed in free recall experiments, where subjects can retrieve the words in any order. Here we analyzed a large dataset from free recall experiments of unrelated words, where short and long words were randomly mixed, and found a seemingly opposite effect: long words are recalled better than the short ones. We show that our recently proposed mechanism of associative retrieval can explain both these observations. Moreover, the direction of the effect depends solely on the way study lists are composed.

Keywords: long term memory, modeling, associative memory, neural network

\section{INTRODUCTION}

Recently we have proposed a mechanism of associative information retrieval that explicitly takes into account longterm neuronal representations of memory items (Romani et al., 2013). One of the basic predictions of the model is the existence of "easy" and "difficult" words. This prediction was verified in our analysis of a large dataset of free recall experiments collected in the lab of Michael Kahana, where we showed that the probability of words to be recalled are consistent between arbitrarily chosen groups of subjects (Katkov et al., submitted). The natural question posed by these observations is what features are predictive for the word difficulty in recall experiments, in particular what if any is the contribution of the word length.

Most of the previous studies of word length effect used lists that were specifically composed of either short or long words. In two previous studies where lists composed of alternating short and long words were used, no word length effect was observed (Hulme et al., 2004; Jalbert et al., 2011). Our current contribution uses free recall paradigm and is based on a much larger dataset than previous studies. We report that when words are selected randomly, irrespective of their length, long words are recalled better than short ones, in a seeming contradiction to classical word length effect in both serial and free recall (Baddeley et al., 1975; Russo and Grammatopoulou, 2003; Tehan and Tolan, 2007; Bhatarah et al., 2009). We provide a possible resolution of this contradiction in the framework of the associative retrieval model of (Romani et al., 2013).

\section{MATERIALS AND METHODS EXPERIMENTAL METHODS}

The data reported in this manuscript were collected in the lab of M. Kahana as part the Penn Electrophysiology of Encoding and Retrieval Study (see Miller et al., 2012 for details of the experiments). Here we analyzed the results from the 141 participants (age 17-30) who completed the first phase of the experiment, consisting of seven experimental sessions. Participants were consented according the University of Pennsylvania's IRB protocol and were compensated for their participation. Each session consisted of 16 lists of 16 words presented one at a time on a computer screen and lasted approximately $1.5 \mathrm{~h}$. Each study list was followed by an immediate free recall test. Words were drawn from a pool of 1638 words. For each list, there was a 1500 ms delay before the first word appeared on the screen. Each item was on the screen for $3000 \mathrm{~ms}$, followed by jittered 800-1200 ms inter-stimulus interval (uniform distribution). After the last item in the list, there was a 1200-1400 ms jittered delay, after which the participant was given $75 \mathrm{~s}$ to attempt to recall any of the just-presented items. All trials were used; intrusions and repetitions were removed from trials.

\section{THE MODEL}

We assume that each word is represented by a randomly chosen population of neurons in the dedicated memory network. We further assume that each retrieved item acts as an internal cue for the next one according to similarity measure between items, which is defined as a the size of the intersection between the corresponding populations (the number of neurons that represent both items). Following (Romani et al., 2013), we consider 
the retrieval process that is directly determined by memory representations of the items, without explicitly simulating network activity. The dynamics of the retrieval is described by a sequence of recalled items. The first one is randomly chosen among the presented ones, and each subsequent recalled item chosen to be the one that has a maximal similarity to the currently recalled one, not counting just "visited" item (Romani et al., 2013). The recall is terminated when the retrieval process enters a cycle and no more items can be retrieved.

To mimic the experimental protocol (see above), we generated $W=1638$ random binary patterns of length $\mathrm{N}:\left\{\xi_{i}^{w}=0 ; 1\right\}$ with $w=1, \ldots, W ; i=1, \ldots, N$ indicates the neurons in the network, such that $\xi_{i}^{w}=1$ if neuron $i$ is participating in the encoding of the memory item $w$. The similarity between items $w$ and $w^{\prime}$ is then computed as $\mathrm{S}^{w w^{\prime}}=\sum_{i=1}^{N} \xi_{i}^{w} \xi_{i}^{w^{\prime}}$. The pattern components for each item were drawn independently with the probability $p_{w}$ of $\xi_{i}^{w}=1$ chosen in the following way: each pattern was arbitrarily assigned a syllabic length $l_{w}=1 \ldots 4$ such that the distribution of $l_{w}$ across the patterns matched the corresponding distribution across the words used in the experiment (five words with syllabic length larger than four were combined with those of length four). For patterns with given $l_{w}$, corresponding $p_{w}$ were equidistantly distributed from $0.02-10^{-3} l_{w}$ to $0.02+10^{-3} l_{w}$. With this choice of pattern statistics, the average number of neurons representing a given item does not depend on its syllabic length, whereas the variance is increasing with syllabic length. The word representations were then fixed throughout the simulated experiment.

For each simulated recall trial, $L=16$ items were chosen for presentation according to two experimental protocols. For the first one, items were selected completely independently, as in the experiment of Kahana. For the second protocol, items with the same $l_{w}$ were randomly selected. Recall process was simulated as in (Romani et al., 2013). The first recalled item was randomly chosen among the presented ones. Subsequent transitions between recalled items were determined by the similarity matrix $S$ between them, each element of which was computed as the number of neurons in the intersection between the corresponding representations: $S^{w w^{\prime}}=\sum_{i=1}^{N} \xi_{i}^{w} \xi_{i}^{w^{\prime}}$. More specifically, the next retrieved item is the one that has the maximal similarity to the currently retrieved one, excluding the item that was retrieved just before the current one. The recall is terminated when the retrieval process enters a cycle and no more items can be retrieved.

\section{RESULTS}

We analyzed a large dataset of free recall experiments performed by 141 subjects with 112 trials per subject. The data is collected in the lab of Michael Kahana. The lists were composed of 16 words randomly selected from a pool of 1638 words. All trials were used; intrusions and repetitions were removed from trials (in total 15792 trials, see Section Methods). For each word, its overall recall probability $\left(P_{\text {rec }}\right)$ was computed as the fraction of trials this word was recalled when it was presented. Figure 1 shows the distribution of $\left(P_{\text {rec }}\right)$ for all words having the given number of syllables (black) aggregated from all trials. The

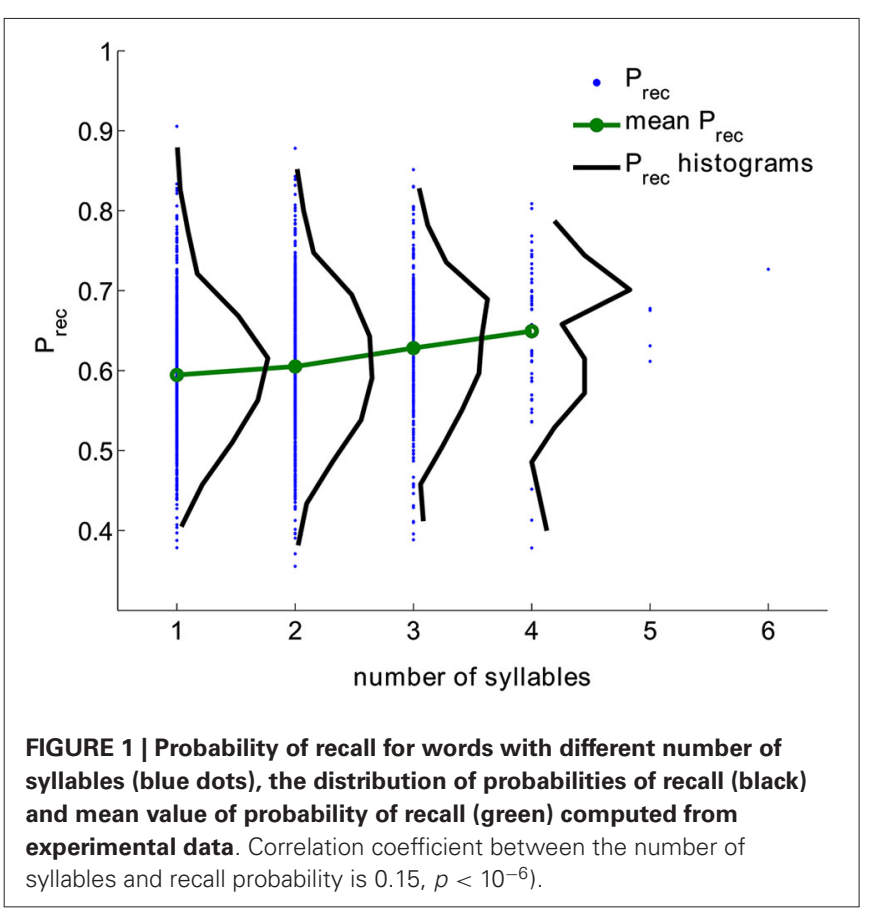

distribution of $P_{\text {rec }}$ is wide for all word lengths. Nevertheless, the mean probability of recall and its variance grow monotonically with the number of syllables (correlation coefficient is 0.15 , $\left.p<10^{-6}\right)$.

This result seemingly contradicts the classical word length effect, where lists of short words were shown to be recalled better than lists of longer words (Baddeley et al., 1975; Russo and Grammatopoulou, 2003; Tehan and Tolan, 2007; Bhatarah et al., 2009). To test whether both these effects may be explained by our proposed retrieval mechanism, we simulated the model imitating experimental paradigms in two conditions-free recall with lists composed from short/long words and random lists (see Methods). The surprising result emerged: the performance in free-recall task depends on the experimental paradigm-in recall of random mixture of unrelated words longer words are statistically easier to recall, whereas in lists composed of words with the fixed number of syllables, shorter words are easier to recall (Figure 2).

Most explanations of classical word length effects assume that total length of presented stimuli is negatively correlated with the number of recalled words. To test whether this statement is supported by the data we computed correlation between the number of syllables in presented lists and the number of recalled words. We found practically no correlation (correlation coefficient is 0.004 and is not significantly different from 0 , $p=0.67)$.

\section{DISCUSSION}

Word length effect, i.e., the observation that lists of short words are recalled better than lists of long words (Baddeley et al., 1975) is considered to be one of the key phenomena in the theories of short-term memory (Campoy, 2011; Jalbert et al., 2011). Here 


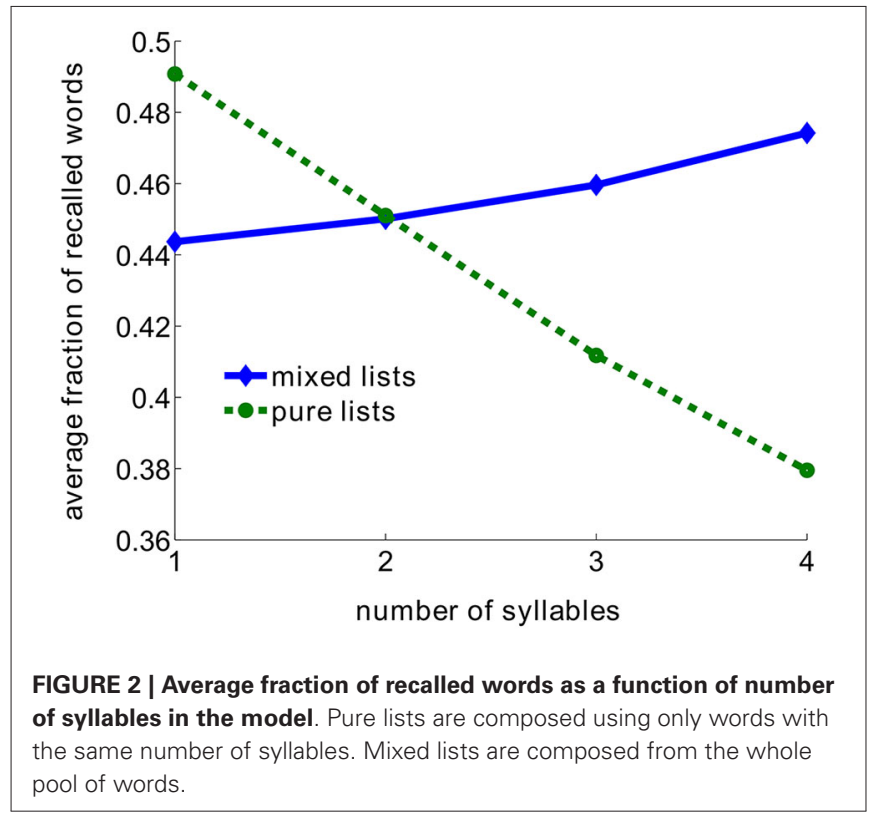

we report that in free recall of unrelated words, where short and long words are randomly mixed, long words have higher recall probability than short ones, in seeming contradiction to the word length effect.

The classical word length effect is traditionally explained by either increased complexity of longer items (Neath and Nairne, 1995), or increased rehearsal time of longer items (Baddeley, 1986, 2003; Page and Norris, 1998; Burgess and Hitch, 1999). The first account suggests that shorter words are generically easier to recall, which is not compatible with our observation. In the second account, more of short words can be rehearsed, due to shorter rehearsal time, and therefore more of them are recalled. This explanation does not specify in what order one would rehearse presented words, but it suggests a negative correlation between the total length of presented items and number of recalled words, whereas no such correlation exists in data.

Here we show that our recently suggested mechanism of associative retrieval can potentially account for both the classical word length effect (which is also present in free recall experiments, see Russo and Grammatopoulou, 2003; Bhatarah et al., 2009) and the opposite length effect in lists of randomly selected words reported in this contribution. In contrast to existing models, long-term neuronal representation of items plays crucial role in our model, and no separate short-term memory mechanism is required. In particular, recall probability of items in random lists is increasing with the size of its representation relative to that of other items, and these items are recalled earlier and suppress the items with smaller representations (Romani et al., 2013). The average recall probability of the whole pool of items is however independent on the average representation size but relates negatively to the variance of representation size across the pool (Katkov et al., submitted). We therefore assumed that longer words do not, on average, have larger representation than shorter ones, but collectively have a higher variance of representation size. This assumption has currently no direct biological justification, but allowed us to reconcile the seeming contradiction between the experimental observations. In particular, it accounts for the classical word length effect, where only words with a given syllabic length are presented, and therefore the variance of representation size increases with syllabic length. In lists with mixed syllabic length, in some trials items with longer syllabic length have largest neuronal representation. When these lists are presented, longer words have larger probability to be recalled, suppressing other items from being recalled, resulting in mild positive correlation between syllabic length of an item and its recall probability.

The results presented in this contribution show that the length of the word is a prominent factor that affects the easiness for it to be recalled. However, we note that recall probabilities still show a wide distribution even for words of given length, indicating that other, yet unknown, word features also contribute to the probability to recall a word.

\section{ACKNOWLEDGMENTS}

We are grateful to M. Kahana for generously sharing the data obtained in his laboratory with us. The lab of Kahana is supported by NIH grant MH55687. Misha Tsodyks is supported by EU FP7 (Grant agreement 604102), Israeli Science Foundation and Foundation Adelis. Sandro Romani is supported by Human Frontier Science Program long-term fellowship.

\section{REFERENCES}

Baddeley, A. D. (1986). Working Memory. Oxford, England: Oxford University Press.

Baddeley, A. D. (2003). Working memory and language: an overview. J. Commun. Disord. 36, 189-208. doi: 10.1016/s0021-9924(03)00019-4

Baddeley, A. D., Thomson, N., and Buchanan, M. (1975). Word length and the structure of short-term memory. J. Verbal Learn. Verbal Behav. 14, 575-589. doi: 10.1016/s0022-5371(75)80045-4

Bhatarah, P., Ward, G., Smith, J., and Hayes, L. (2009). Examining the relationship between free recall and immediate serial recall: similar patterns of rehearsal and similar effects of word length, presentation rate and articulatory suppression. Mem. Cognit. 37, 689-713. doi: 10.3758/MC.37.5. 689

Burgess, N., and Hitch, G. (1999). Memory for serial order: a network model of the phonological loop and its timing. Psychol. Rev. 106, 551-581. doi: 10. 1037//0033-295x.106.3.551

Campoy, G. (2011). Retroactive interference in short-term memory and the wordlength effect. Acta Psychol. (Amst) 138, 135-142. doi: 10.1016/j.actpsy.2011.05. 016

Hulme, C., Suprenant, A. M., Bireta, T. J., Stuart, G., and Neath, I. (2004). Abolishing the word-length effect. J. Exp. Psychol. Learn. Mem. Cogn. 30, 98106. doi: 10.1037/0278-7393.30.1.98

Jalbert, A., Neath, I., Bireta, T. J., and Surprenant, A. M. (2011). When does length cause the word length effect?. J. Exp. Psychol. Learn. Mem. Cogn. 37, 338-353. doi: $10.1037 / \mathrm{a} 0021804$

Miller, J. F., Kahana, M. J., and Weidemann, C. T. (2012). Recall termination in free recall. Mem. Cogn. 40, 540-550. doi: 10.3758/s13421-011-0178-9

Neath, I., and Nairne, J. S. (1995). Word-length effects in immediate memory: overwriting trace decay theory. Psychon. Bull. Rev. 2, 429-441. doi: 10. 3758/bf03210981

Page, M. P. A., and Norris, D. (1998). The primacy model: a new model of immediate serial recall. Psychol. Rev. 105, 761-781. doi: 10.1037//0033-295x.105. 4.761-781 
Romani, S., Pinkoviezky, I., Rubin, A., and Tsodyks, M. (2013). Scaling laws of associative memory retrieval. Neural Comput. 25, 2523-2544. doi: 10 1162/NECO_a_00499

Russo, R., and Grammatopoulou, N. (2003). Word length and articulatory suppression affect short-term and long-term recall tasks. Mem. Cognit. 31, 728-737. doi: 10.3758/bf03196111

Tehan, G., and Tolan, G. A. (2007). Word length effects in long-term memory. J. Mem. Lang. 56, 35-48. doi: 10.1016/j.jml.2006.08.015

Conflict of Interest Statement: The authors declare that the research was conducted in the absence of any commercial or financial relationships that could be construed as a potential conflict of interest.
Received: 27 August 2014; accepted: 26 September 2014; published online: 14 October 2014.

Citation: Katkov M, Romani S and Tsodyks M (2014) Word length effect in free recall of randomly assembled word lists. Front. Comput. Neurosci. 8:129. doi: $10.3389 /$ fncom.2014.00129

This article was submitted to the journal Frontiers in Computational Neuroscience.

Copyright (c) 2014 Katkov, Romani and Tsodyks. This is an open-access article distributed under the terms of the Creative Commons Attribution License (CC BY).

The use, distribution and reproduction in other forums is permitted, provided the original author(s) or licensor are credited and that the original publication in this journal is cited, in accordance with accepted academic practice. No use, distribution or reproduction is permitted which does not comply with these terms. 